\title{
Tulasnein and Podospirone from the Coprophilous Xylariaceous Fungus
}

\section{Podosordaria tulasnei}

Daniela C. Ridderbusch ${ }^{\mathrm{a}}$, Roland W. S. Weber ${ }^{\mathrm{a}, *}$, Timm Anke ${ }^{\mathrm{a}}$, and Olov Sterner ${ }^{\mathrm{b}, *}$

a Department of Biotechnology, University of Kaiserslautern, Paul-Ehrlich-Str. 23,

67663 Kaiserslautern, Germany. E-mail: rwsweber@rhrk.uni-kl.de

b Department of Organic and Bioorganic Chemistry, Lund University, P. O. Box 124,

22100 Lund, Sweden. E-mail: Olov.Sterner@bioorganic.lth.se

* Authors for correspondence and reprint requests

Z. Naturforsch. 59c, 379-383 (2004); received March 17, 2004

Tulasnein (1), a new metabolite with strong antimicrobial and weaker cytotoxic and phytotoxic activity, was isolated from culture filtrates of three strains of the xylariaceous coprophilous fungus Podosordaria tulasnei. The producing strains were identified by their rhizomorphs and by ITS rDNA sequence analysis. A second new metabolite, podospirone (2), was also produced by all three strains whereas the weakly cytotoxic (+)-3,4-anhydroshikimic acid methyl ester (3) was detected in only one strain.

Key words: Podosordaria tulasnei, Podospirone, Tulasnein 